\title{
REGULATION OF NUCLEAR POWER PLANTS A MULTI OBJECTIVE APPROACH
}

\author{
Alok Mishra and Mahesh D. Pandey \\ Institute for Risk Research, University of Waterloo, \\ 200 University Avenue West, Waterloo, \\ Ontario, Canada.
}

\begin{abstract}
The regulation of nuclear power plant is evolving in a direction to embrace imposition of probabilistic safety criteria in the near future. The utilities will not only have to demonstrate that they are operating below a targeted risk level but also to demonstrate that the unavailability of some of the critical safety systems are below a targeted level. It is expected that the risk effective ness of the technical specification and maintenance requirements will be looked into by the utilities not only from risk effective purview but also from cost effective stand point. Thus multi objective optimization of TS\&M requirements is of profound interest to arrive at solutions which may have conflicting objectives. The constraint multi objective optimization of the TS\&M requirements of a Nuclear Power Plant (NPP) based on risk and cost, gives the pareto-optimal solutions, from which the utility can pick its decision variables suiting its interest, which will also satisfy the probabilistic safety criteria. In this paper a multi objective Genetic Algorithm (GA) technique has been used to make a trade-off between risk and cost both at the plant level for Loss of Coolant Accident (LOCA) and Main Steam Line Break (MSLB) as initiating events with an additional intent of meeting the probabilistic safety criteria (PSC).
\end{abstract}

\section{INTRODUCTION}

The Probabilistic Safety Criteria (PSC) has been gaining grounds [1] in several countries and there is an increasing trend in recent years for the regulators to impose requirement for a nuclear power plant utility to demonstrate that a particular safety system/function meets a specific reliability goal, apart from the plant meeting the specified risk level. Thus the primary implication has been for the nuclear regulatory authorities to use Probabilistic safety criteria to evaluate the reliability of specific safety systems and functions as in put to determine if reliability improvements would be justified. In these cases the primary criteria have been derived from top-level goals relating to core melt probability or cost/benefit considerations in which benefit is estimated in terms of averted economic losses resulting from the accident. The requirement of a probabilistic safety criterion in a way guarantees the top-level requirement of a limiting core damage frequency. Thus it is imperative for a regulatory body to prescribe a requirement stating that a nuclear power plant under licensing review should have lower than some level of core damage frequency and additionally some of the critical safety systems should have unavailability lower than some specified value. In such cases the Technical Specification and Maintenance Requirements, which in a way guarantees the safety goals and targets, under goes a multi objective optimization problem with constraints to maintain some of the critical safety systems unavailability within certain specified values.

The development of efficient testing and maintenance (surveillance testing interval, preventive maintenance period, allowed outage time and overhaul period of components) has been traditionally one of the different ways to guarantee high levels of systems availability, which are implemented at NPP through Technical Specification and Maintenance Requirements (TS\&M). Technical specifications (TSs) define the limits and conditions for operating NPPs in a manner that is consistent with the evaluations in the plant's safety analysis report. The general goal of effective acceptable regulations will be to make requirements and activities more risk-effective and less costly by using the risk information to focus better on what is risk-important. The resource versus risk-control effectiveness principles formally enter in optimization problems where the cost or the burden is to be minimized simultaneously with the risk or performance. This relationship applies when TS\&M activities (Surveillance testing Interval (STI), Allowed Outage time (AOT), Preventive Maintenance (PM) frequency) are optimized to minimize both the cost and the unavailability of safety-related systems/ plant risk taking TS\&M-related parameters as the decision variables.

In the past, normally, the resolution of such a kind of optimization problem has been limited to focus only on a single parameter (STI, AOT, PM frequency, etc.) and/or adopting a single optimization criterion (availability, costs, plant risks, etc.) $[2,3,4,5,6,7,8]$. Thus, though the problems posed are multi-objective in nature but they are solved as a single objective problem with constraints. Thus, it is concluded that the earlier models explained as above either lacks in considering all the TS\&M parameters as the decision variables or are not developed to adequately address the plant model. Moreover, none of the models addressed the probabilistic safety criteria, which is likely to be in force in the new regime of regulations.

In this paper a constrained multi objective optimization problem is solved with an objective to minimize cost and core damage frequency and to ensure that some of the safety critical systems have unavailability below a certain specified level. Two postulated initiating events LOCA and MSLB of a PWR plant have been considered for the analysis. The optimization study has been done to decide the surveillance test interval STI), allowed outage time (AOT), the 
preventive maintenance (PM) period and the replacement/ overhaul time of components, such that the risk (CDF due to the initiating event) and the cost of maintaining the required availability of the systems are optimized. A multi objective Genetic Algorithm technique has been used to arrive at the Pareto optimal solution, for optimizing both risk and cost. The unavailability of the high pressure safety injection system, the low pressure safety injection system and the steam generator emergency cool down system are treated as constrained functions to arrive at a solution space which are not only pareto optimal in terms of risk and cost but also satisfies the constraints, meaning restricts the unavailability of the said safety systems below a target value.

\section{PROBLEM FoRMULATION}

The multi-objective constraint optimization of TS\&M will be formulated by adopting the model of Risk and Cost as the objective functions to be minimized and the unavailability function of the safety systems as constraints. TS\&M are represented through appropriate parameters included within the models of risk and cost, which will be adopted as decision variables for the optimization process. The overview of the solution framework is depicted in figure1.

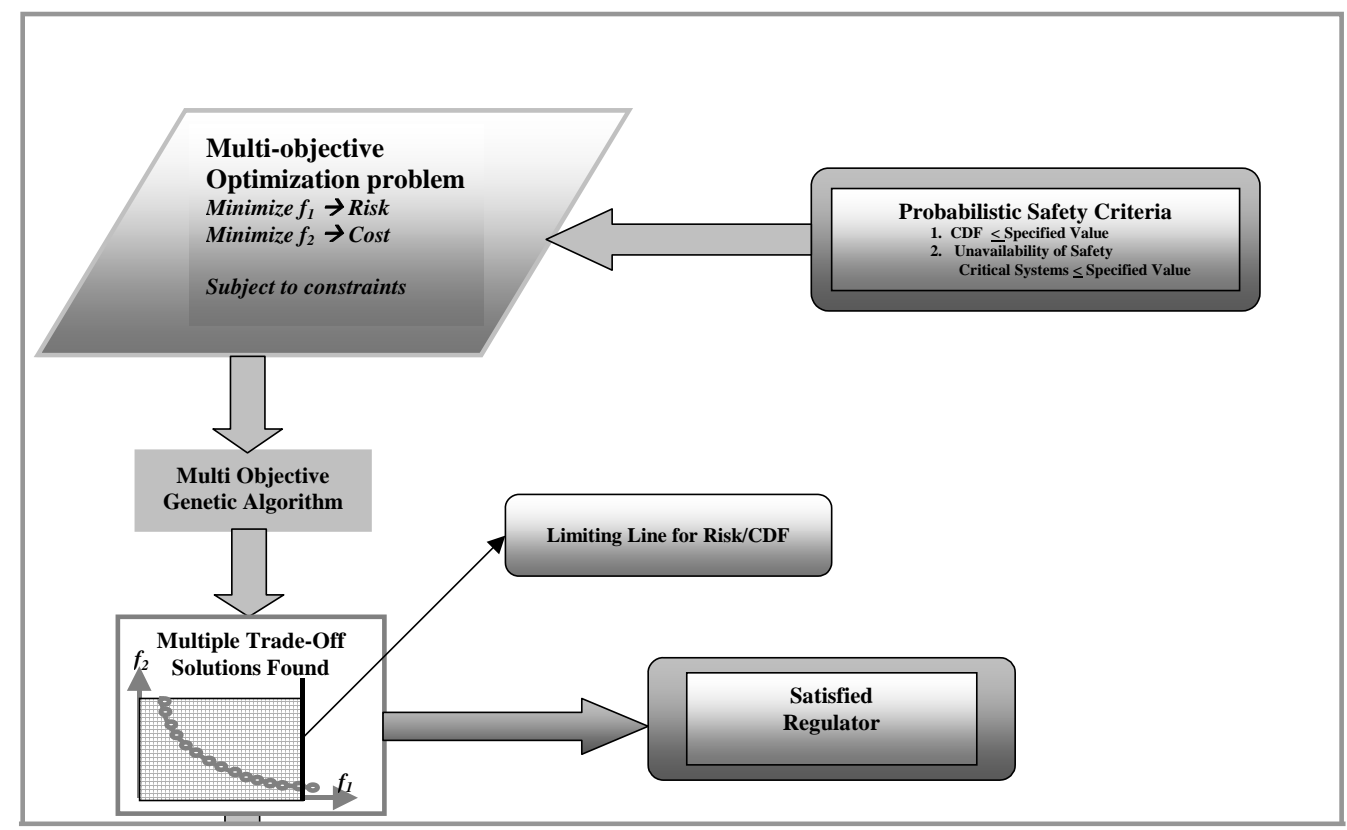

Figure:1 Overview of multi objective optimization for meeting

probabilistic safety criteria

\section{Risk Function}

In a nuclear power plant the occurrence of a postulated Initiating Event (IE) with the unavailability of the mitigating safety systems may lead to Core Damage (CD) sate. The core damage frequency can be defined as the risk associated with a nuclear power plant. Thus the risk associated with a particular initiating event 'r'can be defined as

$$
\text { Risk }_{\mathrm{r}}=\text { I.E. } \text { frequency }_{\mathrm{r}} \times \sum_{p=1}^{q}[\text { C.D. Event Sequences }]_{p}
$$

Where, p extents from 1to q, is the identity of the core damage (CD) event sequences.

The CD event sequences results due to the occurrence of an I.E. and unavailability of a single or a group of mitigating safety systems. Thus the Risk $\mathrm{r}_{\mathrm{r}}$ can be defined as a function of the I.E. frequency and the unavailability of the mitigating systems i.e.

$$
\text { Risk }_{\mathrm{r}}=I . \text { E. frequency }_{\mathrm{r}} \times\left[U_{a}(x) \times U_{b}(x) \ldots \ldots+U_{a}(x) \times U_{c}(x) \ldots \ldots+\ldots \ldots .\right]
$$

$U(x)$ is the unavailability of the mitigating safety system and sub-script $a, b, c$ are for different mitigating safety systems. 
The system unavailability model (which can also be modeled as a risk function at a system level ) is often formulated into the Probabilistic Risk Assessment (PRA) adopting the rare event approximation, which is an upper bound, as follows [9]:

$$
U(x) \approx \sum_{j} \prod_{k} u_{j k}(x)
$$

Where the sum in $j$ extents to the number of Minimal Cut sets (MCS) after the reduction of the system structure function and the product in $k$ extents to the number of basic events relevant to the corresponding MCS. In addition, $u_{j k}(x)$ is the unavailability associated with the basic event $k$ belonging to MCS $j$, which quantifies a given unavailability state of a safety component that depends on the vector of decision variables $x$. Consequently, quantification of the different contributions to the component unavailability is needed to derive the final risk function using Eq.(1-3), which, in turn, will be used as one of the objective functions. Thus by using only Eq.(3), we can write the risk function (unavailability) at the system level and using Eq.(2), we can formulate the risk function at the plant level for a particular initiating event.

The unavailability contributions of a component normally in standby are divided into two categories: 1) unavailability due to random failures, named the reliability effect, and 2) unavailability due to testing and maintenance downtimes, named the downtime effect. In modern NPPs some of the active safety system functions are combined with normal operation functions, with a view to increase functional reliability. In such cases the design provides full-fillment of safety functions with minimum number of changes of valves position. Here, in our approach, we have adopted Martorell's approach [7] for the standby system and further extend the Martorell's approach to extent the model for the operating systems and components [10,11]. Thus, the basic component unavailability contributions considering the TS\&M parameters for the risk function calculation are depicted in Table 1.

Table 1

Formulation of basic component unavailability contributions

\begin{tabular}{|c|c|c|c|}
\hline \multirow[t]{2}{*}{ No. } & \multirow[t]{2}{*}{ Description } & \multicolumn{2}{|l|}{ Formulations } \\
\hline & & Standby system & Operating system \\
\hline 1 & Random failures & $\rho+\frac{1}{2} \lambda_{1}^{*} T$ & $\lambda_{0}^{k} T_{m}$ \\
\hline 2 & Testing & $t T$ & \\
\hline 3 & Corrective maintenance & $\left(\frac{1}{7}\right)\left(\rho+\lambda_{5}^{*} T\right)\left(1-e^{-(D / \mu)}\right) \mu$ & $\lambda_{0}^{*} \mu\left(1-e^{-(D / \mu)}\right)$ \\
\hline 4 & Preventive maintenance & $\begin{array}{l}m / M \\
\lambda *=\lambda+\frac{1}{2} \alpha M(\psi(z))^{2}\left[1+(1-\varepsilon)\left(\frac{L}{M}-3\right)\right]\end{array}$ & $m / M$ \\
\hline
\end{tabular}

$\rho$ : demand failure probability; $\lambda_{\mathrm{g}}:$ standby failure rate; $\lambda_{0}:$ operating failure rate; $D$ : allowed outage time; $T$ : surveillance tes interval; $t$ : testing time; $M$ : preventive maintenance pericd; $m$ : preventive maintenance time; $\mu$ : mean time to repair; $\alpha$ : linear aging factor, $\psi(z)$ : working condition; $\varepsilon$ : maintenance effectiveness and $L$ : overhaul period.

There are several postulated initiating event which has potential of causing core damage if the required mitigating systems fail to perform its intended function. Thus the cumulative Core Damage Frequency (CDF) due to all such events can be defined as the risk associated with the operation of a Nuclear Power Plant (NPP).

$$
\sum \text { Risk }=\sum_{m=1}^{n}\left\{[\text { I.E. frequency }]_{m} \times \sum_{p=1}^{q}[\text { C.D.EventSequences }]_{p}\right\}
$$

Where m extents from 1 to $\mathrm{n}$, is the identity of the initiating event and p extents from 1to $\mathrm{q}$, is the identity of the core damage (CD) event sequences.

\section{Cost Function}

The relevant costs in analyzing TS\&M optimization of safety-related equipment include the contributions of on-line systems as well as the standby components, which undertake surveillance testing with period $\mathrm{T}$, preventive maintenance with period $\mathrm{M}$ and corrective maintenance to restore their operability after a failure has been discovered during a test. Consequently, summing up the corresponding cost contributions of the relevant components to yield the cost model:

$$
C(x)=\sum_{i} c_{i}(x)
$$

which will be used as the other objective function in the optimization problem. These models are presented in detail in Martorell's approach [7] and extended to accommodate the online systems by Mishra, et.al., [10,11], are summarized here in Table-2. 
Table 2

Formulation of basic cost contributions

\begin{tabular}{|c|c|c|c|}
\hline \multirow[t]{2}{*}{ No. } & \multirow[t]{2}{*}{ Description } & \multicolumn{2}{|l|}{ Formulations } \\
\hline & & Standby system & Operating system \\
\hline 1 & Testing & $\left(\frac{t}{7}\right) C_{\mathrm{rx}}$ & \\
\hline 2 & Preventive maintenance & $\left(\frac{\mathrm{n}}{\mathrm{M}}\right) \mathrm{C}_{\mathrm{mm}}$ & $\left(\frac{\pi}{m}\right) \cdot C_{h \pi}$ \\
\hline 3 & Corrective maintenance & $\left(\frac{1}{7}\right)\left(\rho+\lambda_{1}^{*} T\right) \mu\left(1-e^{-(D / \mu)}\right) C_{\mathrm{lc}}$ & $\lambda_{0}^{*} \mu\left(1-e^{-(D / \mu)}\right) C_{\mathrm{hC}}$ \\
\hline 4 & Plant shut-down (repoir time exceeds AOT) & $\left(\frac{1}{T}\right)\left(\rho+\lambda_{3}^{*} T\right)\left(e^{-(D / \mu)}\right) C_{u}$ & $\lambda_{0}^{*}\left(e^{-(D / \mu)}\right) C_{u}$ \\
\hline 5 & Overhaul maintenance & $\left(\frac{1}{L}\right) C_{r}$ & $\left(\frac{1}{I}\right) C_{t}$ \\
\hline
\end{tabular}

$C_{\mathrm{ht}}$ : hourly cost for surveillance testing: $C_{\mathrm{hm}}$ : hourly cost for preventive maintenance; $C_{\mathrm{h}}$ : hourly cost for corrective maintenance; $C_{\mathbf{a}}$ : cost for plant shutdown: $C_{\mathrm{r}}$ cost for overhaul.

\section{Constraint Functions}

The multi objective optimization problem of risk and cost is intended to be solved with the constraint of unavailability of the critical safety systems below a targeted value. Thus the constraint function is defined as

$$
1.0 \times 10^{-4}-U(x) \geq 0
$$

It implies that the unavailability of the mitigating system will be less than $10^{-4} \mathrm{yr} / \mathrm{yr}$ (which is used as a target value).

\section{EXAMPLE OF APPLICATIONS}

In a nuclear power plant two of the most important postulated initiating events are (i) loss of coolant accident (LOCA) and (ii) main steam line break (MSLB). In the case of LOCA, borated cooling water is injected into the reactor core to remove the decay heat and to preserve core integrity by the emergency core cooling system (active and passive systems). Similarly, in case of MSLB the core cooling is ensured through the secondary side by actuation of steam generator emergency cool-down (SG-ECD) system and passive heat removal system (PHRS).

In a typical advanced pressurized water reactor the emergency core cooling system comprises of four sub-systems known as (1) high pressure active safety injection (HPSI) system, (2) high pressure 1st stage passive safety injection system (HA-I), (3) low pressure 2nd stage passive safety injection system (HA-II) and (4) low pressure active safety injection (LPSI) system. The secondary side emergency cooling system comprises of steam generator emergency cooldown (SG-ECD) system and passive heat removal system (PHRS). These are all quadruplet-train safety systems. Failure of these safety systems during LOCA or MSLB as the case may be will lead to core damage, and hence, the availability of these systems is the main concerns for the safety management and regulation of a nuclear power plant.

The following cases are analysed with a view to satisfy the probabilistic safety criteria.

i. Loss of Coolant Accident (LOCA) considered with the constraint function for restricting the unavailability of the High Pressure Safety Injection (HPSI) system and Low Pressure Safety Injection (LPSI) system limited to a value lower than $1 * 10^{-4} \mathrm{yr} / \mathrm{yr}$.

ii. Main Steam Line Break (MSLB) considered with the constraint function for restricting the unavailability of the Steam Generator Emergency Cool Down (SG-ECD) system limited to a value lower than $1 * 10^{-4} \mathrm{yr} / \mathrm{yr}$.

iii. LOCA and MSLB considered together with the constraint function for restricting the unavailability of the HPSI and SG-ECD systems limited to a value lower than $1 * 10^{-4} \mathrm{yr} / \mathrm{yr}$.

The objective functions of risk and cost with the constraint functions for the $3^{\text {rd }}$ case as indicated above are derived using the equations (3), (4) and (5) and has the following form

$$
\begin{gathered}
\text { Risk }_{\text {LOCA }+M S L B}(x)=\left[\operatorname{Risk}_{L O C A}(x)+\operatorname{Risk}_{M S L B}(x)\right] \\
\text { Cost }_{\text {plant }}(x)=\operatorname{Cost}_{L O C A}(x) \cup \operatorname{Cost}_{M S L B}(x) \\
\text { Subject to }: \\
1.0 \times 10^{-4}-U(x)_{H P S I} \geq 0 \\
1.0 \times 10^{-4}-U(x)_{S G-E C D} \geq 0
\end{gathered}
$$

The constraint multi objective optimization is also performed for the risk and cost optimization for the risk and associated cost for the initiating events of LOCA and MSLB individually, with constraint on unavailability restrictions on HPSI, LPSI and SG-ECD systems respectively for meeting the probabilistic safety criteria.

\section{System Modeling and Data}

The system modeling consists of the active and passive safety injection systems and the underlying safety support systems. A train is assumed to consist of the front-line safety system and its support system. For the active systems the surveillance test interval, the allowed outage time and the preventive maintenance period are train specific and are chosen same for all the components in a train. It is also considered that during the preventive maintenance the entire train 
with all its components is under preventive maintenance. The over-haul periods of the components are for the individual components. For a passive system the surveillance test interval and the preventive maintenance are to coincide with the annual scheduled plant outage, and hence these values have to be forced as once per year.

The success/failure criteria of the above systems as defined by the safety analysis report are used and the fault tree analysis for generation of the Minimal Cut Sets (MCS) of these systems have been done using the computer software "Risk Spectrum" [12]. The cut-off value for the Minimal Cut Sets (MCS) for the unavailability of the High Pressure Safety Injection System, the Low Pressure Injection System and the Steam Generator Emergency Cool-down System are taken as $3.85 * 10^{-11}, 3.78 * 10^{-11}$ and $8.33 * 10^{-10}$ respectively.

The obtained Minimal Cut-sets have been used to define the two objective function based on risk and cost. The various component data required for the fault tree analysis are considered as per the Probabilistic Safety Analysis for Nuclear Power Plant guide lines. For considering the effect of ageing for all the components Proportional Age Reduction (PAR) model [7] is used.

The component unavailability and the cost parameters as required in the formulations for the risk and cost functions modeling are given in Reference [7,10].

\section{Decision Variables}

Any solution to the optimization problem can be encoded using the following array of decision variables: $X=\{T, D$, $\left.M, L_{1-n}\right\}$. In this analysis it is considered that the $T$ i.e. Surveillance test interval, $D$ i.e. Allowed Outage Time and $M$ i.e. Preventive Maintenance Period. $L_{1-n}$ is the over-haul time of components in the mitigating front-line systems and support systems. The above decision variables can be bounded by lower and upper bound to get a solution in the desired feasible domain. The bounding conditions lower bound and upper bound for the decision variables are considered as follows $T(168,4032), D(72,720), M(4000,16000)$ and $L_{1-n}(8000,80000)$, all the above mentioned values are in hours. The current TS\&M requirement for the decision variables are as follows: $X=\left\{T=672, D=168, M=8000, L_{1-n}=32000\right\}$.

\section{Genetic Algorithm for Multi OBJective Optimization}

Evolutionary Algorithms (EAs) mimic natural evolutionary principles to constitute search and optimization procedures in a variety of ways. Genetic Algorithms (GAs) are search and optimization algorithms inspired by the principles of natural evolutionary principles. Conceived by John Holland in the early sixties, GAs have enjoyed a number of applications and developments. One of the niches of GA's application domains is in the field of multi-objective optimization, where instead of one objective the problem is faced with number of conflicting objectives.

Over the past decade, a number of multi-objective evolutionary algorithms (MOEAs) have been suggested [14,15]. The primary reason for this is their ability to find multiple pareto-optimal solutions in a single simulation. In this study one such multi-objective EA - a real coded "Non-dominated Sorting Genetic Algorithm with Elitism (NSGA-II) $[15,16,17]$ is used and is discussed in the following sections.

\section{NSGA-II Solution Algorithm}

The Non-dominated Sorting Genetic Algorithm or NSGA-II procedure [17] for finding multiple pareto-optimal solutions in a multi-objective optimization problem has the following three features:

- It emphasis the non-dominated solutions

- It uses an explicit diversity preserving mechanism

- It uses an elitist principle

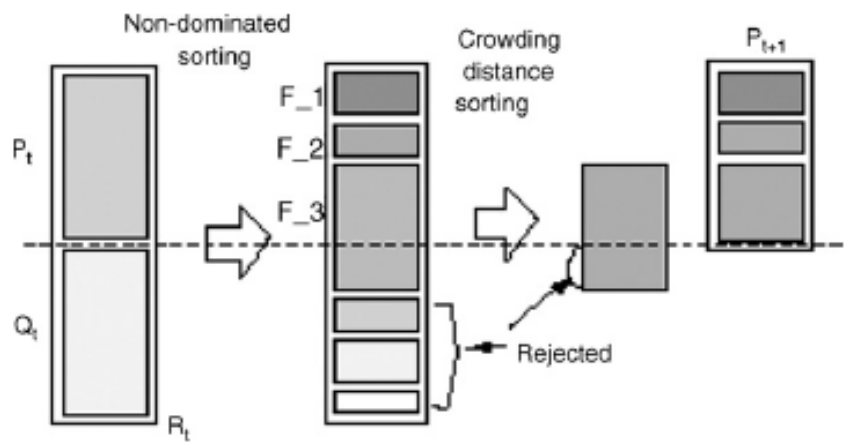

Fig. 2. Schematic of MOGA procedure.

In NSGA-II, the initial population $\left(P_{t}\right)$ of size $N$ is randomly created within the feasible boundary for each decision variables. Then the off-spring population $Q_{t}$ is created by using the parent population $P_{t}$. The parent and the child population are then combined to form $R_{t}$ of size $2 N$. Then, a non-dominated sorting is used to classify the entire 
population $R_{t}$. Although this requires more effort compared to performing a non-dominated sorting on $Q_{t}$ alone, it allows a global non-domination check among both the parent and child solutions. Meaning the elitism in the solution set is preserved. Once the non-dominated sorting is over, the new population is filled by solutions of different non-dominated fronts, one at a time. The filling starts with the best non-dominated front and continues with the solutions of the second non-dominated front, followed by the third non-dominated front, and so on. Since the over-all size of $R_{t}$ is $2 N$, not all fronts may be accommodated in $\mathrm{N}$ slots available in new population. All fronts which could not be accommodated are simply deleted. When the last front is being considered there may exist, more solutions in the last front than the remaining slots in the new population. Then instead of arbitrarily discarding some members from the last front, it would be wise to use niching strategy to choose the members of the last front, which reside in the least crowded region in that front. The above scheme is depicted in figure-2.

\section{Crowded Tournament Selection Operator}

A solution I wins a tournament with solution $\mathrm{j}$ if any of the following conditions are true:

$>$ If solution I has a better rank, that is, $r_{i}<r_{j}$.

$>$ If they have the same rank but solution I has a better crowding distance than solution $j$, that is, $r_{i}=r_{j}$ and $\mathrm{d}_{\mathrm{i}}>\mathrm{d}_{\mathrm{j}}$.

The first condition makes sure that the chosen solution lies on a better non-dominated front. And the second condition resolves the tie of both solutions being on the same non-dominated front by deciding on their crowded distance. The one residing in a less crowded area (with a large crowding distance) wins.

\section{Crowding Distance}

To get an estimate of the density of solutions surrounding a particular solution I in the population, we take the average distance of two solutions on either side of solution I along each of the objectives. This quantity $d_{i}$ (the crowding distance) serves as an estimate of the perimeter of the cuboid formed by using the nearest neighbors as the vertices. The crowding distance $\mathrm{d}_{\mathrm{i}}$ can be calculated by several ways, in this study a crowding distance metric [16] , which requires $O(M N \log N)$ computations is used. The other methods have $O\left(M N^{2}\right)$ complexity.

\section{Cross-over Operator}

Since the design variables take any real number, a real-coded genetic algorithm is used in this study. In a real coded GA, variables are not coded in binary strings instead GA operators are directly applied on the real numbers. Although any selection operators can be used, specialized cross-over and mutation operators are used to effectively create offspring solutions from parent solutions. In this study, we use Simulated Binary Crossover (SBX) and a parameter-based mutation operator [18]. The cross-over rate is fixed at 0.9 and the mutation rate is fixed at 0.01 . All the simulations are run for 500 generations.

\section{Constraint Tournament Selection operator}

The Constraint Tournament Selection Method is a penalty parameter less constraint handling approach [16,19]. Here, two solutions are picked from the population and the better solution is chosen. In the presence of constraints, each solution can be either feasible or infeasible. Thus, there may be at most three situations: (i) both solutions are feasible, (ii) one is feasible and other is not, and (iii) both are feasible. For a single objective optimization, a simple rule for each case is used:

Case (i) : Choose the solution with the better objective function value.

Case (ii) : Choose the feasible solution

Case (iii) : Choose the solution with smaller over all constraint violation.

In the context of multi-objective optimization, the latter two cases can be used as they are presented, but the difficulty arises with the first case, as the confusion arises so as which objective function is to be considered. In such cases the concept of domination comes to the rescue. When both the solutions are feasible, it is checked if the solutions belong to the separate non-dominated fronts. In such event the one with the better non-dominated front is chosen. If they belong to the same non-dominated front then the diversity preservation technique is used to resolve the tie. Since maintaining diversity is another goal in multi objective optimization, we can choose the one, which belongs to the least crowded region in that non-dominated set. Thus the following constrain-domination condition for any two solutions $x^{(i)}$ and $x^{(j)}$.

A solution $x^{(i)}$ is said to be "constrain-dominate" a solution $x^{(j)}$ ( or $\left.x^{(i)} \preceq_{c} x^{(j)}\right)$, if any of the following condition are true:

- Solution $x^{(i)}$ is feasible and solution $x^{(j)}$ is not.

- Solutions $x^{(i)}$ and $x^{(j)}$ are both infeasible, but solution $x^{(i)}$ has a smaller constraint violation.

- Solutions $x^{(i)}$ and $x^{(j)}$ are feasible and solution $x^{(i)}$ dominates solution $x^{(j)}$.

Thus among a population of solutions, the set of non-constrain-dominated are those that are not constrain-dominated by any member of the population. By using the definition of constraint domination, we define the generic constrained tournament selection operator as follows:

- Given two solutions $x^{(i)}$ and $x^{(j)}$, choose solution $x^{(i)}$, if any of the following conditions are true: 
- Solution $x^{(i)}$ belongs to a better non-constrain-dominated set.

- Solution $x^{(i)}$ and $x^{(j)}$ belong to the same non-constrain-dominated set, but solution $x^{(i)}$ resides in a less crowded region based on a crowding distance metric.

\section{RESULTS AND DISCUSSION}

The constraint multi objective optimization at the plant level has been studied for the risk vs. the cost/hr of maintaining the mitigating safety system. The decision variables are the surveillance test interval, the allowed outage time, the preventive maintenance period and the overhaul time of various components appearing in the minimal cut sets (MCS). The Pareto-optimal solutions of risk and cost are given in the figure: 3, 4 and 5 for the LOCA, MSLB and LOCA + MSLB cases respectively.

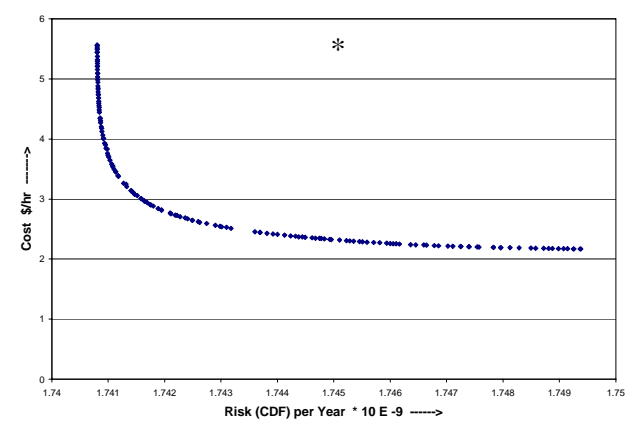

Figure: 3 Pareto optimal Solutions for LOCA meeting Probabilistic Safety Criteria

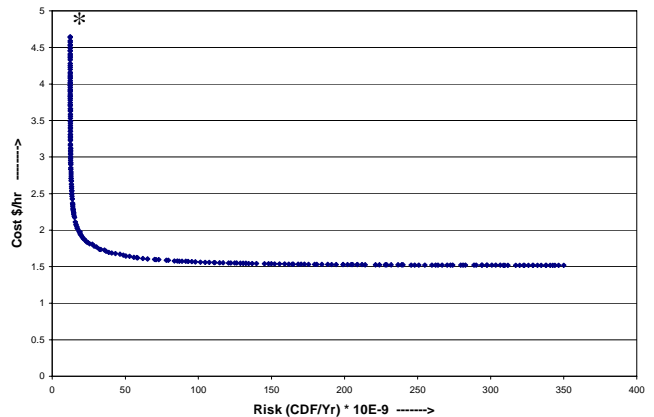

Figure: 4 Pareto optimal Solutions for MSLB meeting Probabilistic Safety Criteria

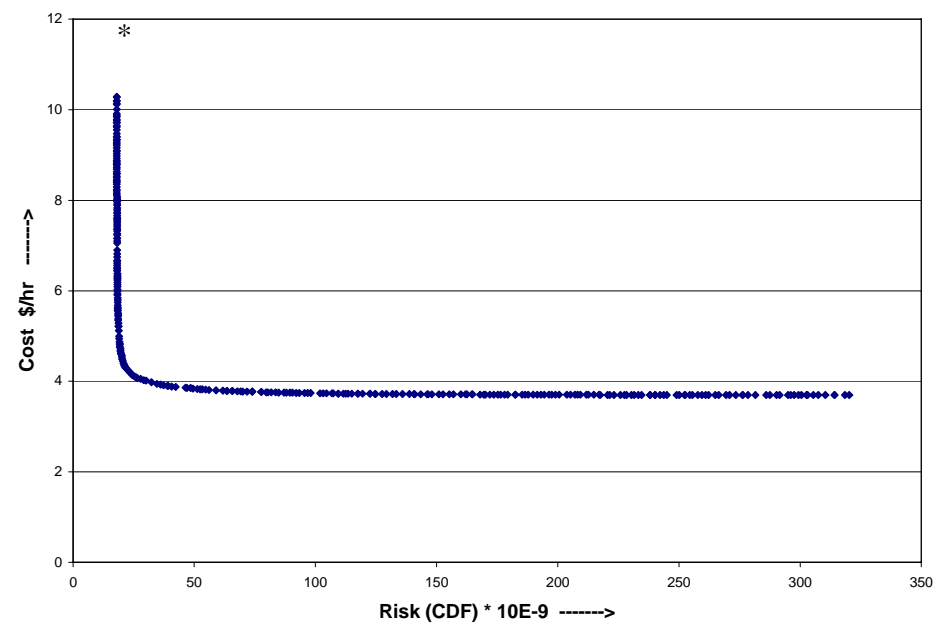

Figure: 5 Pareto optimal Solutions for LOCA \& MSLB Combination

Meeting Probabilistic Safety Criteria

* indicates the risk and cost profile at the current level of regulation in figure (3-5)

The figures also depict the risk and cost profile at the current level of regulation. In the $1^{\text {st }}$ case the Pareto optimal solutions of risk and cost for Loss of Coolant Accident (figure: 3) ensures that the unavailability of the high pressure injection (HPSI) system and low pressure safety injection system (LPSI) are below $1.0 \times 10^{-4} \mathrm{yr} / \mathrm{yr}$. Similarly for the $2^{\text {nd }}$ case the Pareto optimal solutions of risk and cost for the Main Steam Line Break Accident (figure: 4) ensures that the unavailability of the Steam generator Emergency Cool Down (SG-ECD) system is below $1.0 \times 10^{-4} \mathrm{yr} / \mathrm{yr}$. The figure: 5 depicts the Pareto optimal solution of risk and cost for LOCA and MSLB, considered together. It ensures that the unavailability of both HPSI and SG-ECD system individually are below $1.0 \times 10^{-4} \mathrm{yr} / \mathrm{yr}$. Thus if we choose a particular cut-off value of core damage frequency the solution space to the left of that point will give us the alternatives to meet the CDF requirement as well as the probabilistic safety criteria. Choosing the best solution is a matter of debate and discussion in all multi objective optimization processes. To arrive at a particular solution to adopt higher level 
information are required. One of the alternatives is to look at the knee point where the cost to risk ratio is marginalized meaning the increase in cost gives very insignificant benefit in risk reduction. In future the decision space of such solutions can be considered for implementation at the utility. The passive systems are not considered as there are restrictions in altering the TS\&M requirements for them because of their configuration in the systems. Such systems necessarily have to be tested during the outages only and are specified for testing and maintenance during the annual outage.

In all these above cases we can see that the risk level is satisfactory from regulatory stand point however, the risk and cost profile of the plant at the current level of regulation is away from the pareto optimal front. Thus the regulation of the decision variables are not risk consistent and scope exists to improve the risk and cost profile of the plant and at the same time the probabilistic safety criteria can be met.

\section{CONCLUSION}

There is an increasing trend in recent years for the regulators to impose requirement of Probabilistic Safety Criteria (PSC) on a nuclear power plant utility to demonstrate that a particular safety system/function meets a specific reliability goal, apart from the plant meeting the specified risk level. The multi objective optimization of the TS\&M requirements of a NPP based on risk and cost with appropriate constraints, gives the pareto-optimal solutions, from which the utility can pick its decision variables suiting its interest and can also satisfy the probabilistic safety criteria. All of the optimized TS\&M parameters in the example of application represent practical alternatives that can be implemented in a NPP under a real situation. The decision variables of STI, AOT, PM and the over-haul period for different systems and equipment chosen from the pareto-optimal front will be risk consistent. The NSGA-II algorithm constraint tournament selection method has proved to be very efficient in arriving at the optimal solutions in a reasonably small number of generations.

\section{REFERENCES}

1. IAEA-TECDOC-529: Probabilistic safety criteria at the safety function/system level, Vienna, (1989).

2. Painton, L., and Campbell. J., Genetic algorithms in optimization of system reliability. IEEE Transactions on Reliability 442 (1995), p. 172.

3. Vaurio, J.K., Optimization of test and maintenance intervals based on risk and cost. Rel. Engng and System Safety 49 (1995), p. 23.

4. Harunuzzaman, M., and Aldemir, T., Optimization of standby safety system maintenance schedules in nuclear power plants. Nuclear Technology 113 (1996), p. 354

5. Muñoz, A., Martorell, S., and Serradell, V., Numerical absolute and constrained optimization of maintenance based on risk and cost criteria using genetic algorithms. Proceedings of Advances in Safety and Reliability, ESREL'97. Lisbon, p. 1749.

6. Levitin, G., and Lisnianski, A., Join redundancy and maintenance optimization for multistage series-parallel systems. Rel. Engng and System Safety 64 (1999), p. 33.

7. Martorell, S., Sanchez, A., Carlos, S., and Serradell, V., Simultaneous and Multi Criteria Optimization of TS requirements and Maintenance at NPPs, Annals of Nuclear energy 29 (2002), p. 147-168.

8. Yang, J., Sung, T..and Jin, Y., Optimization of the surveillance test interval of the safety systems at the plant level. Nuclear Technology 132 (2000), P. 352.

9. Henley J, Kumamoto H. reliability engineering and risk assessment. Englewood Cliffs, NJ:Printice-Hall;1981.

10. Mishra, A., 2006. Regulation of Nuclear Power Plant a multi-Objective Approach, Ph.D. Thesis, IIT Bombay.

11. Mishra, A., Patwardhan, A., and Verma, A.K., Safety management in NPPs using an evolutionary algorithm technique, Nuclear Engineering and Design, 2007.

12. Risk Spectrum Manual

13. Martorell, S., Serradell, V., Verdu, G., Fragio, R., Sanchez, A., Martinez, M.L., Gil, S., and Izquierdo, J.A., Maintenance optimization by using PSA results in the RCM context. 1996,IAEA-TECDOC-873, 257.

14. Horn, J.,Nafploltis, N., and Goldberg, D.E., (1994). A niched pareto genetic algorithm for multi-objective optimization. Michalewicz, Z, editor, proceedings of the $1^{\text {st }}$ IEEE Conference on Evolutionary Computation, $\mathrm{P}$ 82-87, Piscataway, Newjersy.

15. Srinivas, N., and Deb, K., Multi-objective function optimization using non-dominated sorting genetic algorithms. Evolutionary Computation, 2(3), 1995,221-248.

16. Deb, K., Multiobjective optimization using evolutionary algorithms. Wiley, Chlchester, UK.

17. Deb, K., Agarwal, S., Pratap, A., and Meyarivan, T., A fast and elitist multi-objective genetic algorithm: NSGAII, IEEE Transactions on Evolutionary Computation, 6(2), 2002, 182-197.

18. Deb, K., and Agarwal, R.B., Simulated binary crossover for continuous search space, Complex Systems 9(1995), 115-148.

19. Deb, K., Pratap, A., and Meyarivan, T., Constrained test problems for multi-objective evolutionary optimization. Proceedings of the First International conference on evolutionary Multi-Criteria Optimizaton (EMO-2001), p. 284-298, (2001). 